\title{
Wire Drawing Effect on Microstructural and Textural Evolution in Medium Carbon Steel Wires
}

\section{NEBBAR Mohamed Chaouki ${ }^{1,3, \mathrm{a}^{*}}$, ZIDANI Mosbah ${ }^{1,2, \mathrm{~b}}$, MESSAOUDI Salim¹, ABID Taher ${ }^{4}$, KISRANE-BOUZIDI Ahmed ${ }^{1}$ and BAUDIN Thierry ${ }^{5}$}

\author{
${ }^{1}$ Energetic and Materials Engineering Laboratory-University of Biskra-Algeria \\ ${ }^{2}$ Faculty of Technology, University of Batna 2-Algeria
}

${ }^{3}$ Scientific and Technical Research Center in Physico-Chemical Analyzes (CRAPC)-Algeria ${ }^{4}$ TREFISOUD, El-Eulma-Sétif-Algéria

5ICMMO, SP2M, Paris-Sud University, Paris-Saclay University, UMR CNRS 8182, build.410, 91405 ORSAY, France.

a*nebbar.mchaouki@gmail.com, ㄹm.zidani@univ-batna2.dz, csalim.messaoudi@univ-biskra.dz, dabidtah@yahoo.fr, ea.kisrane-bouzidi@univ-biskra.dz

\begin{abstract}
This study was mainly oriented on the evolution of the crystallographic texture as a function of the deformation resulting from the industrial wire drawing process. This, in fact, will make it possible to establish a relationship between the microstructure and the crystallographic texture in the medium-carbon steel wires obtained by industrial wire drawing process and used in the manufacture of spring mattresses in order to minimize the loss of material and to satisfy the users of this product. During this study, a medium-carbon steel wires was characterized by two analytical techniques. The scanning electron microscopy (SEM) to monitor the microstructure evolution and the electron backscatter diffraction (EBSD) for the crystallographic texture analysis. The EBSD results are processed with OIM (Orientation Imaging Microscopy) analysis software.
\end{abstract}

Keywords: Medium-carbon steel, Wire drawing, Microstructure, Texture, EBSD, OIM Analysis 


\section{Introduction}

Carbon steels remain the most used materials in drawing industries because of their excellent priceperformance ratio. They offer a wide range of usage properties deriving from their numerous microstructure and texture variants. The microstructural behavior of these steels present themselves as a powerful solution to adapt them to the many implementation processes and the various service requests. This explains the huge amount of research being done on the microstructure of carbon steels in general $[1,2,3,4]$.

The progressive anisotropy of the crystallographic texture or of the elongation of the grains (morphological texture) induced in the material by wire drawing as a consequence of significant changes at the microstructural level. Thus, although it is clear that this deformation process improves the microstructural modifications can produce an anisotropic microstructure [5]. Embury and Fisher [6] and $\mathrm{Li}$ et al [7] studied the evolution of the microstructure and crystallographic texture of a pearlitic steel wires during wire drawing process and described the development of the fibrous structure (morphological texture) and a crystallographic texture of fiber <110>//WDD (where WDD is the wire drawing direction parallel to the wire axis (WA)).

In this work, wire drawing of D45-2 medium-carbon steel wires for producing springs, were studied in longitudinal section to follow the evolutions of the microstructure, crystallographic texture and mechanical properties (Hardness (HV), Tensile strength (Rm), Elongation (A\%) and torsion number) to have the correlation between microstructural and mechanical properties of D45-2 medium-carbon steel wires. 


\section{Material}

The present study was carried out on medium carbon steel wires provided by TREFISOUD JSC., Algeria. Chemical composition and mechanical properties of wire rod are presented in Table 1.

Table 1. Chemical composition (wt \%) and mechanical properties of D45-2 medium-carbon steel wire rod $(\varnothing 5.5 \mathrm{~mm})$.

\begin{tabular}{|c|c|c|c|c|c|c|c|}
\hline \multicolumn{7}{|c|}{ Chemical composition } \\
\hline $\mathbf{C}$ & $\mathbf{M n}$ & $\mathbf{S i}$ & $\mathbf{P}$ & $\mathbf{S}$ & $\mathbf{C r}$ & $\mathbf{N i}$ & $\mathbf{C u}$ \\
\hline 0.465 & 0.653 & 0.152 & 0.009 & 0.013 & 0.069 & 0.039 & 0.054 \\
\hline \multicolumn{8}{|c|}{ Mechanical properties } \\
\hline HV & \multicolumn{3}{|c|}{ Rm (MPa) } & \multicolumn{3}{c|}{ Elongation A\% } \\
\hline 205 & \multicolumn{3}{|c|}{785.83} & \multicolumn{3}{c|}{22.5} \\
\hline
\end{tabular}

D45-2 steel is an non alloyed steel with $0.45 \%$ (average) carbon content and is intended for the wire drawing process for special applications (Fig. 1) [8].

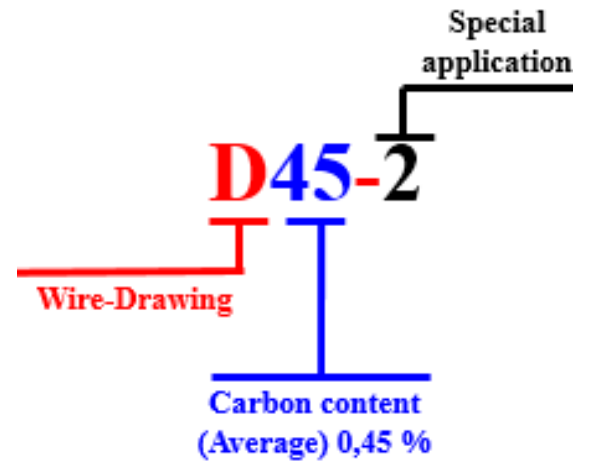

Fig. 1. Standardized designation of D45-2 steel [8].

Sampling is carried out in such a way that structural deformation due to heating during cutting should be avoided. The preferred length is $15 \mathrm{~mm}$. The wires diameter and deformation rates $(\mathcal{E})$ are shown in the Figure 2.

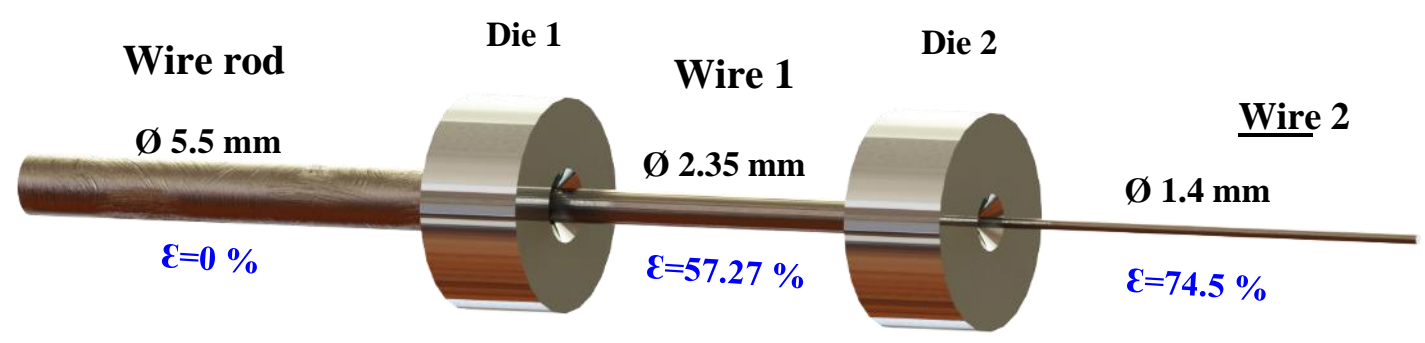

Fig. 2. Studied samples of D45-2 medium-carbon steel wires.

For observation of the microstructure by SE microscopy, all the samples were subjected to mechanical polishing performed different granulometry of SiC papers (P100 to P4000) with oil-based diamond. After the mechanical polishing, the samples were chemically etched with Nital $3 \%$ to reveal microstructure. For the crystallographic texture characterization, the samples were subjected to mechanical polishing on $\mathrm{SiC}$ paper followed by $3 \mu \mathrm{m}$ and $1 \mu \mathrm{m}$ diamond polishing and then electro-polishing to remove the mechanical polishing effect. 


\section{Characterization Techniques}

The evolution of the microstructure and the crystallographic texture in D45-2 medium-carbon steel wires were characterized by scanning electron microscopy FEI quanta 250, electron backscatterdiffraction (TSL EDAX) in the scanning electron microscopy Zeiss 940. The data was processed by OIMTM software.

When by focusing an electron beam on a grain which one wishes to know the crystallographic orientation, the electrons backscattered in Bragg incidence with the reflecting crystallographic planes will diffract according to two diffraction cones for each reflecting plane (Fig.3). The intersection of the diffraction cones with a detector screen forms hyperbolas resembling lines. The set of lines constitutes the Kikuchi diagram.

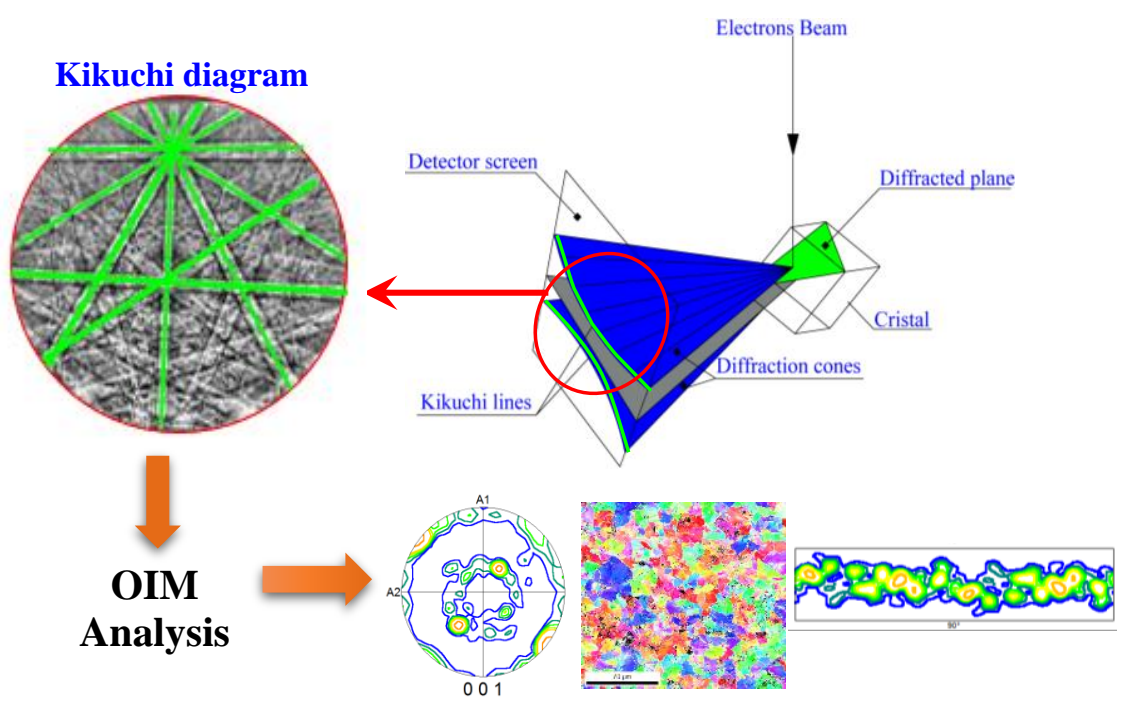

Fig.3. Basic principle of EBSD [9] 


\section{Results and Discussion}

\section{SEM characterization}

Figure 4 presents a SEM micrograph of the D45-2 wire rod. We can notice that the microstructure is composed of two phases (ferrite $(\mathrm{F})$ and pearlite $(\mathrm{P})$ ) observed in the figure 4-B. Each perlite colony (PC) has its own lamellar direction. The cementite lamellae are presented by the light color and the ferrite by the dark color. Two morphologies of perlite can be observed, one with straight lamellae (SPL) predominant and the other with curled lamellae (CPL) (Fig.4-A). The overall microstructure is homogeneous without any sign of plastic deformation and the equiaxed form of the grains with a random crystallographic texture and orientations of the cementite lamellae.
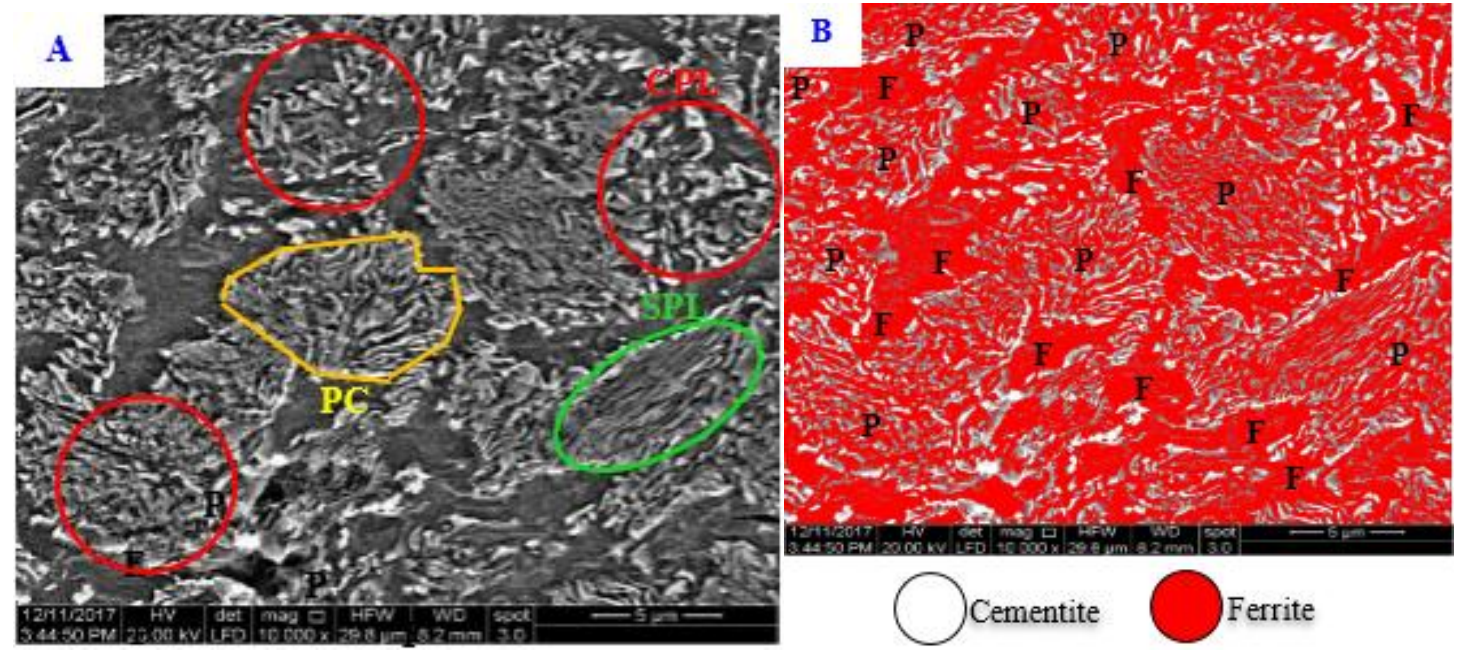

Fig.4. SEM micrograph of the longitudinal section of D45-2 steel wire rod (Ø $5.5 \mathrm{~mm})$ A) SEM micrograph, B) Illustration of the compounds (processed by ImageJ)

In the same way, we present on figure 5, the SEM images relating to two different deformation rates by drawing $(57.27 \%$ and $74.5 \%)$ of steel D45-2. We can notice three types of cementite lamellaeare clearly identifiable: aligned with the WDD folded and sheared at $57.27 \%$ deformation rate and aligned with the DD direction at $74.5 \%$. The microstructure has become fibrous and the grains are strongly elongated in the direction of wire drawing DD.
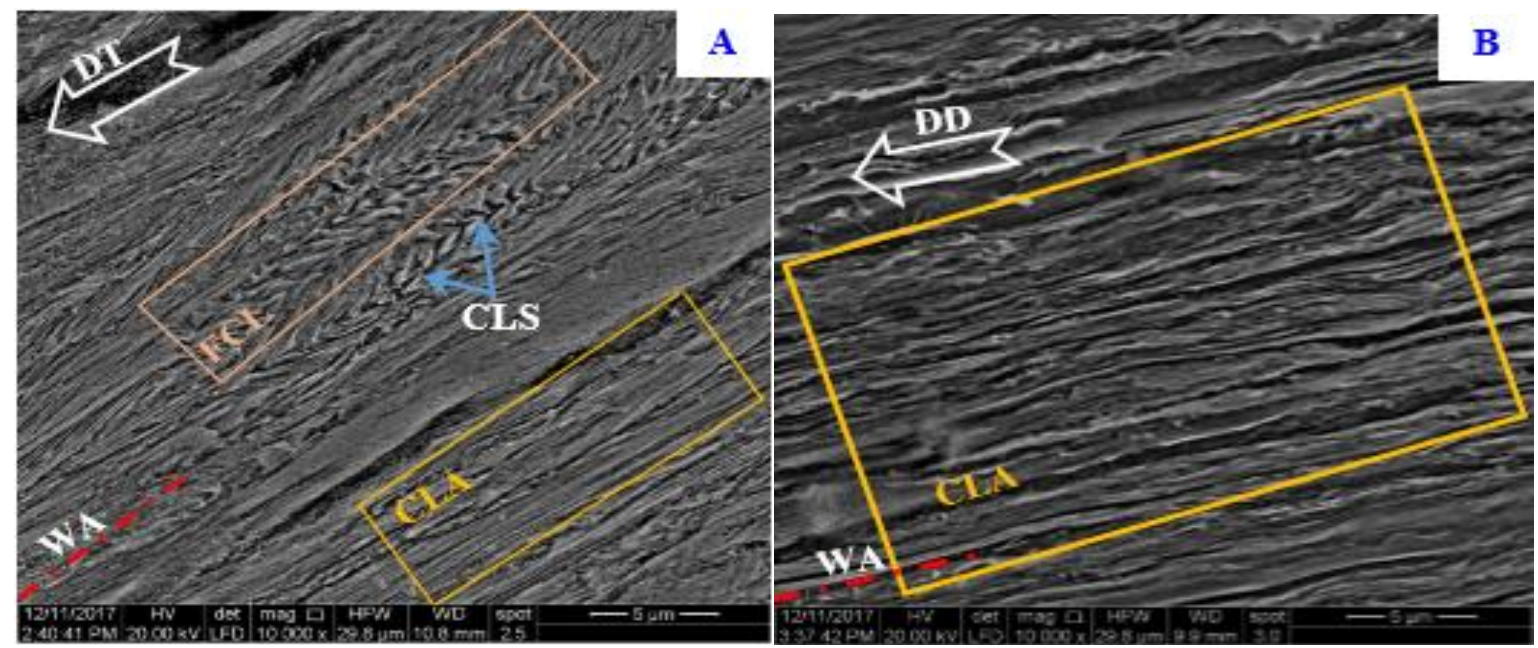

Fig. 5. SEM micrographs of the longitudinal section of the drawn steel wire D45-2 drawn. a) $\varepsilon=57.27 \%$ and b) $\varepsilon=74.5 \%$. 


\section{EBSD Characterization}

First of all, we can see on the poles figure of drawn wires (Fig.6) the presence of the fiber texture $\langle 110\rangle$ parallel to the direction A1 (drawing direction) [10], this fiber texture is developed during deformation by industrial wire drawing and reinforced with the maximum intensities of 15.378 for $\varepsilon$ $=57.27 \%$ and 14.603 for $\varepsilon=74.5 \%$.
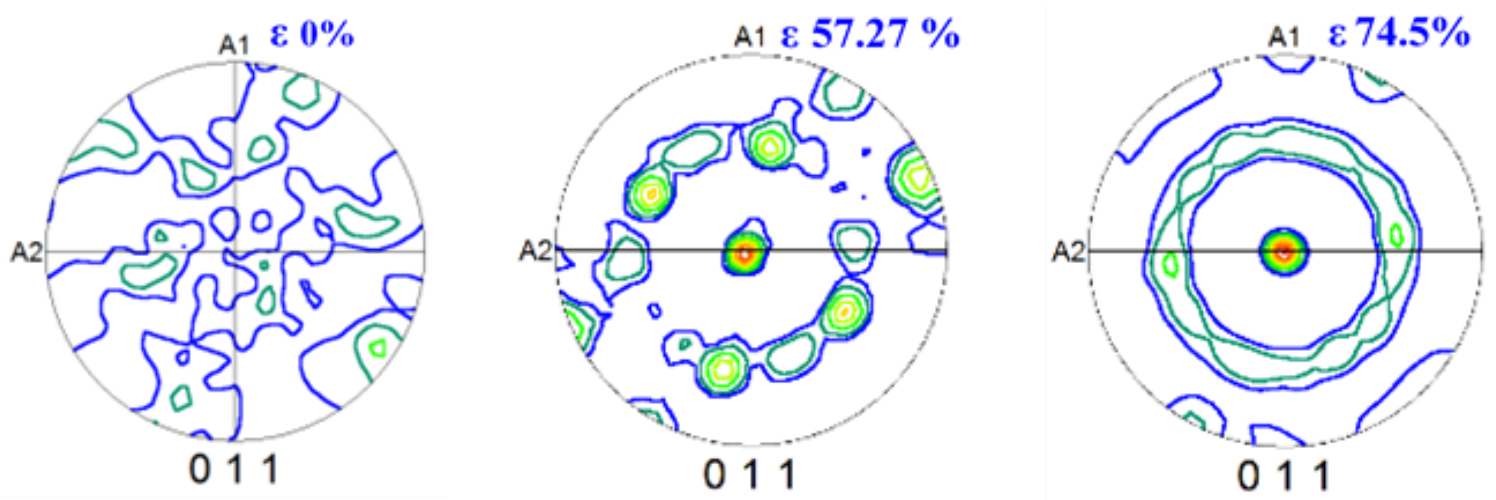

Fig. 6. Poles figure $\{110\}$ of D45-2 steel wires.

In Figure 7 (ODF), only a development of the $<110>/ /$ WDD fiber texture appears at $\varepsilon=57.27$ $\%$. This fiber is in the majority and reinforced at $\varepsilon=74.5 \%$.

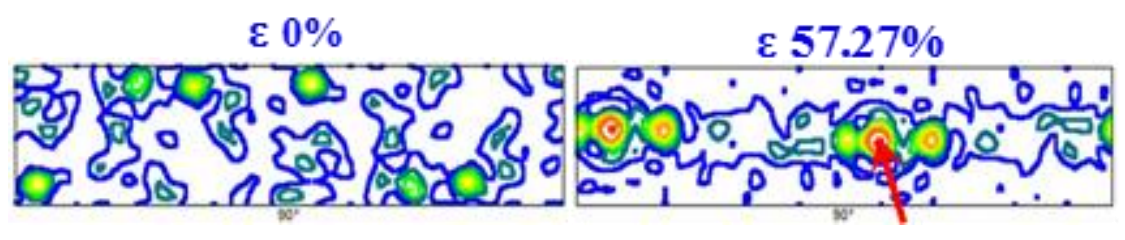

$<110>/ /$ WDD $\varepsilon 74.5 \%$

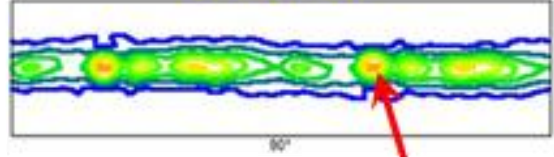

$<110>/ /$ WDD

Fig.7. Orientation distribution functions (ODF) of D45-2 steel wires. 


\section{Mechanical Properties}

The Vickers hardness of D45-2 medium-carbon steel increases with the deformation rate by wire drawing and reach a value of 310 for $\varepsilon=57.27 \%$ and 417 for $\varepsilon=74.5 \%$. This hardening is due to the generation of additional dislocations in the steel structure and the refining of inter-lamellar spacing during $[11,12]$.

Figure 8 shows the effect of deformation rate by wire drawing on the tensile strength, elongation (Fig.8-a) and number of twists before failure-t/f (Fig8-b) as a function of deformation rate of D45-2 medium-carbon steel wires.
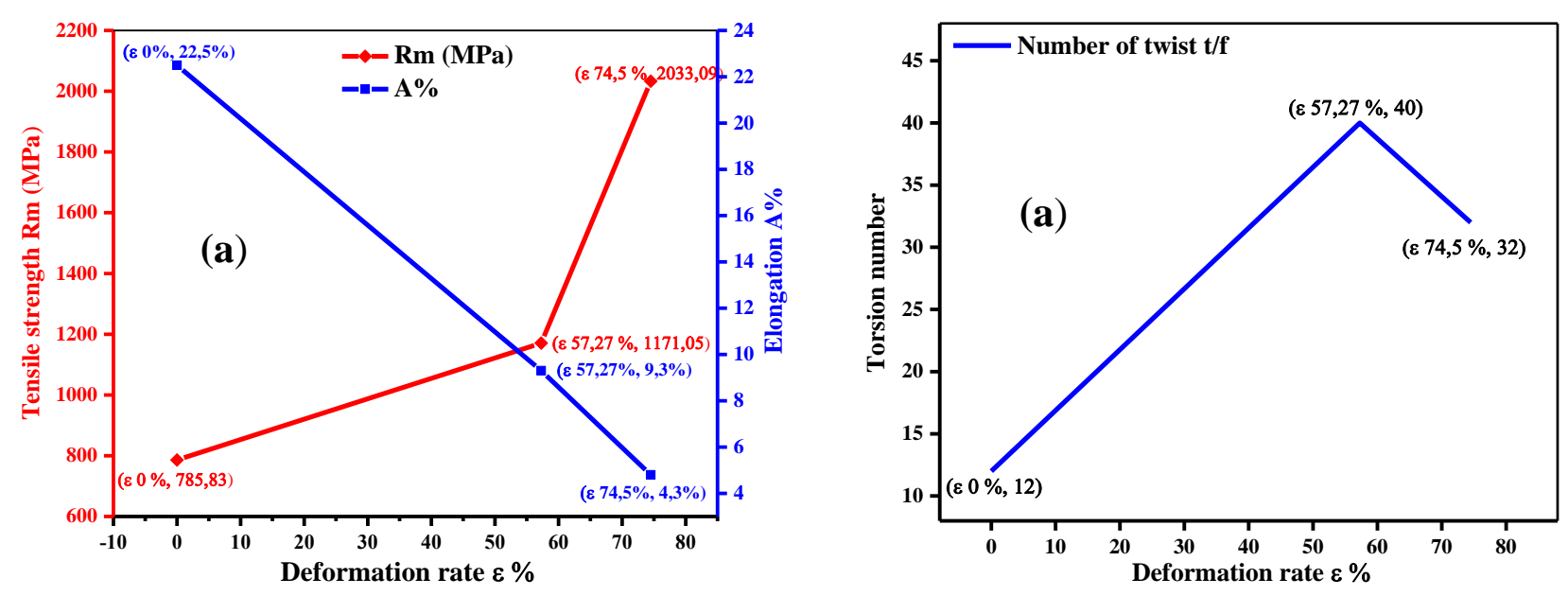

Fig. 8. Mechanical properties of D45-2 medium-carbon steel wires. a) Tensile strength and Elongation, b) Number of twist before failure.

Figures 8 (a) show that tensile strength increases with the deformation rate while elongation decreases significantly. Before drawing, the wire rod has a tensile strength of around 785.83 MPa. The tensile strength of drawn wires achieved 1171.05 MPa and 2033.09 MPa for $57.27 \%$ and 74.5 $\%$ deformation rates, respectively. The number of twists before failure-t/f increases then decreases at $\varepsilon=74.5 \%$. This decrease due to the loss of ductility caused by wire drawing at this deformation rate [13].

\section{Conclusion}

Scanning electron microscopy allowed us to analyze the grains morphology. During wire drawing, the cementite lamellae orient towards the wire drawing direction and the microstructure with a morphological texture parallel to the wire axis. Two different forms of cementite lamellae are observed: folded (FCL) and aligned cementite lamellae (CLA).

EBSD analysis has shown us the evolution of the crystallographic texture as a function of the deformation rate. The development of a fiber texture $<110\rangle$ comes from the drawing effect.

Mechanical tests applied to D45-2 steel wires show that increasing of hardness and tensile strength with decreasing of elongation as a function of the deformation rate. These changes due to grain refinement and the accumulation of crystal defects and increased dislocation density caused by wire drawing. The number of twists before failure $-t / f$ increases then decreases. This decrease due to the loss of ductility of wire at at $\varepsilon=74.5 \%$. 


\section{References}

[1] M.C. Nebbar, M. Zidani, T. Djimaoui, T. Abid, H.Farh, T. Ziar, A.L. Helbert, F. Brisset, T.Baudin. (2019). Microstructural evolutions and mechanical properties of drawn medium carbon steel wire. International Journal of Engineering Research in Africa, 41.1, 1-7.

[2] T. Djimaoui, M. Zidani, M.C. Nebbar, T. Abid, H.Farh, A.L. Helbert, F. Brisset, T.Baudin. (2018). Study of Microstructural and Mechanical Behavior of Mild Steel Wires Cold Drawn at TREFISOUD. International Journal of Engineering Research in Africa, 36, 53-59.

[3] L. Zhou, Y. Zhao, F. Fang. (2014). Effect of reserved texture on mechanical properties of cold drawn pearlitic steel wire. Advanced Materials Research, 936, 1948-1952.

[4] X. Zhang, A. Godfrey, N. Hansen, X. Huang. Hierarchical structures in cold-drawn pearlitic steel wire.(2014). Acta Materialia, 61, 4898-4909.

[5] J.Toribio. Structural integrity of progressively cold-drawn pearlitic steels: From Raffaello Sanzio to Vincent van Gogh. (2017). Procedia Structural Integrity, 3, 3-10.

[6] J.D. Embury and R.M Fisher.(1966). The structure and properties of drawn pearlite. Acta Metallurgica, 14, 147-159.

[7] Y.J.Li, P.Choi, S.Goto, C.Borchers, D.B.Raabe, R.Kirchheim. (2012). Evolution of strength and microstructure during annealing of heavily cold-drawn $6.3 \mathrm{GPa}$ hypereutectoid pearlitic steel wire. Acta Materialia, 60, 4005-4016.

[8] DIN 17140-1 Grade D 45-2.

[9] M.C.Nebbar. Etude de l'évolution microstructurale, texturale et de comportement mécanique des fils en acier mi-dur tréfilés industriellement. (2020).Doctoral thesis, University of BiskraAlgeria.

[10] M.Zidani, S.Messaoudi, T.Baudin, D.Solas, M. H. Mathon. (2010). Deformation textures in wire drawn perlitic steel. International Journal of Material Forming, 3, 7-11.

[11] N.A.Raji and O.O.Oluwole. (2013) Mechanical properties of cold-drawn low carbon steel for nail manufacture: experimental observation.Research Journal of Applied Sciences, Engineering and Technology, 5, 118-122.

[12] C.C.Robert, B.Forfert, B.bolle, J.J.Fundenberger, A.Tidu. (2008). Influence of torsion deformation on microstructure of cold -drawn pearlitic steel wire. Journal of Materials Science, 43, 1241-1248.

[13] M.Zelin. Microstructure evolution in pearlitic steels during wire drawing. Acta Materialia, 50, 4431-4447.

\section{Acknowledgments}

The authors would like to thank the industrial partners of TREFISOUD Company and S. Amirouche for his technical support. 